\title{
Word Level Stress and Lexical Processing in 17-Month-Old Infants
}

\author{
Jennifer Campbell \\ Department of Psychology \\ The University of British Columbia \\ Susan Graham, and Suzanne Curtin \\ Department of Psychology \\ University of Calgary
}

\begin{abstract}
Detailed representations enable infants to distinguish words from one another and more easily recognize new words. We examined whether 17-month-old infants encode word stress in their familiar word representations. In Experiment 1, infants were presented with pairs of familiar objects while hearing a target label either properly pronounced with the correct stress (e.g., baby /'berbi/) or mis-pronounced with the incorrect stress pattern (e.g., baby /ber'bi/). Infants mapped both the correctly stressed and mis-stressed labels to the target objects; however, they were slower to fixate the target when hearing the mis-stressed label. In Experiment 2, we examined whether infants appreciate that stress has a nonproductive role in English (i.e., altering the stress of a word does not typically signal a change in word meaning) by presenting infants with a familiar object paired with a novel object while hearing either correctly stressed or mis-stressed familiar words (Experiment 2). Here, infants mapped the correctly stressed label to the familiar object but did not map the misstressed label reliably to either the target or distractor objects. These findings suggest that word stress impacts the processing of familiar words, and infants have burgeoning knowledge that altering the stress pattern of a familiar word does not reliably signal a new referent.
\end{abstract}

During the first year of life, infants acquire knowledge about the sound structure of the language(s) they are learning. Learning about the phonological properties of their language, including native sound categories and sound combinations, can help infants learn words (for a review see Curtin \& Zamuner, 2014). For example, by 12 months of age, infants use their phonological knowledge to judge and accept well-formed word forms as possible object labels but reject isolated speech sounds, communicative sounds, and phonotactically illegal forms (MacKenzie, Graham, \& Curtin, 2011;

Correspondence should be sent to Suzanne Curtin, Department of Psychology, University of Calgary, 2500 University Drive NW, Calgary, AB T2N 1N4 Canada. E-mail: scurtin@ucalgary.ca 
MacKenzie, Curtin, \& Graham, 2012). At this age, infants will also use word stress-a prominent phonological property - to map novel words to objects (Curtin, 2009). Word stress, however, is not a phonological property that typically disambiguates between object labels in English. Thus, it may not impact recognition of familiar words. Across two experiments, we examined whether 17-month-old infants encode word stress in their familiar word representations (Experiment 1), and appreciate that stress has a nonproductive role in English (i.e., that altering the stress of a familiar word does not typically signal a change in word meaning; Experiment 2).

In English, word stress and grammatical class are correlated such that the majority of disyllabic nouns have a trochaic (strong-weak) stress pattern, and the majority of disyllabic verbs have an iambic (weak-strong) pattern (Kelly \& Bock, 1988). In languages that are thought to assign stress by rule, such as English, stress information is not considered part of the lexical representation (Lahiri \& Marslen-Wilson, 1991; Studdert-Kennedy, 1976). Instead, a set of rules exist for assigning stress to English words (Chomsky \& Halle, 1968) and these rules are stored separately from the phonological representation of the word (Halle, 1998). As a result, the representations of lexical items are considered to be relatively abstract.

Word stress is realized acoustically when a syllable has higher f0 (pitch), has greater intensity (louder) and has longer vowel duration relative to the surrounding syllables within the word (Cutler, 2004). English is a quantity-sensitive language (Hayes, 1995). That is, while the syllable within the word that is stressed has these acoustic properties, vowel reduction in adjacent syllables can occur. Research has demonstrated that adult listeners are sensitive to these acoustic properties; that is, adults detect the mis-stressed familiar words, and this sensitivity is greater when mis-stressing is accompanied by vowel reduction (Cutler \& Clifton, 1984). Indeed, vowel quality is weighted more than other cues to lexical stress including f0, duration, and intensity by English speakers (Zhang \& Francis, 2010). While vowel reduction is an important part of adults' recognition of lexical stress, even when forms contain full vowels, mis-stressing hinders adult recognition of familiar words (Cutler \& Clifton, 1984). Thus, lexical stress, in and of itself, may be useful for adults, and perhaps infants, in differentiating words in the lexicon.

Around 7 months of age, infants begin demonstrating sensitivity to word stress by attending to and using the native language stress patterns to segment words from the speech stream (Curtin, Mintz, \& Christiansen, 2005; Polka \& Sundara, 2003; Polka, Sundara, \& Blue, 2002). By 9 months, English infants show a preference for listening to the dominant stress pattern of English (Echols, Crowhurst, \& Childers, 1997; Jusczyk, Cutler, \& Redanz, 1993) and rely more on stress cues for word segmentation than other forms of cues (e.g., transitional properties; Johnson \& Jusczyk, 2001; Thiessen \& Saffran, 2003). Infants' ability to attend to stress and use it to segment the speech signal does not require them to encode lexical stress in their word forms; however, there is some early evidence that they might encode lexical stress into their word forms. For example, although 11-month-old English-learning infants do not show an overall listening preference for properly stressed familiar words over mis-stressed familiar words in a head turn preference procedure, they have a delayed response to mis-stressed words (Vihman, Nakai, DePaolis, \& Halle, 2004). In this experiment, infants preferred the properly stressed words over the mis-stressed words in the first half of the trials, suggesting an influence of stress on recognition. By the second half of the experiment, however, infants did not show a preference between the two trial types. When 36- 
month-old Dutch toddlers are shown two side-by-side images of familiar objects and are presented with disyllabic labels that either have correct stress or incorrect stress, their fixation times for the incorrect stress is lower than when they hear the correct stress (Van Alphen, de Bree, Fikkert, \& Wijnen, 2007). Together, these findings support the claim that infants are sensitive to word stress and that stress may be part of the lexical representation of familiar words.

If infants are storing lexical stress in their familiar word representations, it may be because lexical stress can be used to distinguish words from one another. In English, only a handful of words are primarily distinguished by word stress (indicated here by an accent on the stressed vowel), with word stress typically differentiating nouns (e.g., ínsult, récord, próduce, cónflict, óbject, présent, próject) from verbs (e.g., insúlt, recórd, prodúce, conflíct, objéct, presént, projéct). Word stress, however, is not a productive property of the English language. That is, when a new word is needed or introduced in the language, it is unlikely that altering the word stress of an already existing form would be considered. Thus, while word stress is salient and useful for determining grammatical class, it is not a productive property of the language used for creating new object labels. It remains unclear, however, whether infants treat stress as a productive or necessary part of a familiar word's representation as an object label.

Although no research to date has examined infants' understanding of the nonproductive nature of stress in familiar words, there is evidence that young infants treat word stress as a productive property in their novel word-referent mappings. That is, 12-month-olds treat trisyllabic words that differ only in their stress patterns as distinct words, each mapping on to different objects (Curtin, 2009), while at the same time detecting incorrect stress in a newly learned label for an object (Curtin, 2010, 2011). By 16 months, infants use lexical stress to guide novel verb learning and selectively map iambic word forms to actions, but do not map trochaic word forms to the same actions (Campbell, Mihalicz, Theissen, \& Curtin, 2018; Curtin, Campbell, \& Hufnagle, 2012), suggesting some burgeoning understanding about grammatical class based on word stress.

In the following experiments, we ask whether 17-month-olds encode lexical stress in their familiar word representations and whether they appreciate the nonproductive nature of lexical stress. Infants of this age have demonstrated that they have detailed phonetic representations of their familiar words when tested with vowel and consonant mispronunciations (e.g., Swingley \& Aslin, 2000). Based on this previous work, we predict that infants will store lexical stress in their familiar word representations similar to how they encode other phonetic information. However, lexical stress is unique from most other phonetic information in that it is nonproductive in English. That is, lexical stress is not used to distinguish a novel object-label from a known one. In two experiments, we explore whether infants' early word representations include lexical stress (Experiment 1) and whether infants appreciate the nonproductive nature of lexical stress (Experiment 2).

\section{EXPERIMENT 1}

The goal of Experiment 1 was to examine whether 17-month-old infants encode stress in their familiar word representations. Using a split screen design, we displayed a target object (e.g., an apple) paired with a familiar distractor (e.g., a bottle) while the 
target word was presented. Across trials, familiar disyllabic words were presented twice, once with the correct initial stress (trochaic; e.g., apple /'æpel/) and once with the mis-stressed final (iambic $/ \mathfrak{x} p \varepsilon l /$ ) form. If infants encode stress in their familiar word representations, they should show greater fixations to the target when the words are properly stressed versus mis-stressed and be slower to arrive at the target referent when the words are mis-stressed.

\section{Participants}

Twenty-five 17-month-old infants $(M=17$ months 14 days, $S D=9$ days, range: 17 months 0 days to 17 months 28 days) were included in the final sample. All infants came from homes wherein English was the primary spoken language. While parental education data and socioeconomic status data were not collected, samples collected from this site are predominately White and broadly come from middle to upper socioeconomic status backgrounds. An additional 15 infants were tested and were removed from the analyses due to fussiness $(N=6)$, parental inference $(N=2)$, experimenter error $(N=4)$, and failure to achieve eye-tracking calibration $(N=3)$. We further removed six infants based on their eye-tracking data. Three of these infants had insufficient fixation data recorded in the study, and three of these infants preferred the incorrect object on at least half of our filler trials (please see the "Coding and Data Preparation" section for details on these criteria). This study was conducted according to guidelines laid down in the Declaration of Helsinki, with written informed consent obtained from a parent or guardian for each child before any assessment or data collection. All procedures involving human subjects in this study were approved by the Conjoint Faculties Research Ethics Board (Approval \#REB15-2495) at the University of Calgary.

\section{Auditory stimuli}

Auditory stimuli were recorded by a female native-English speaker in a quiet environment using infant-directed speech. The following six distractor words were recorded with natural pronunciation: banana, bunny, chicken, dinosaur, bottle, and butterfly. The following six disyllabic words were used as target words: apple, baby, flower, kitten, puppy, and teddy. Each target word was presented in two forms, one with the appropriate trochaic stress and one with inappropriate iambic stress, for a total of 12 stress test trials. For example, flower was recorded with trochaic stress as in /'flowə $\mathrm{a} /$ and with iambic stress as in /flow'ə $\mathrm{a} /$. To document the stress patterns for each word, the first and second syllable were compared on pitch, intensity, and duration. As shown in Table 1, the stressed syllables in the trochaic tokens were significantly higher than unstressed syllables in pitch $(t(5)=10.74, p<.001$, Cohen's $d=3.26)$ and intensity $(t$ $(5)=5.09, p<.01$, Cohen's $d=1.73)$. The unstressed syllables in the trochaic tokens were significantly longer than the stressed syllables $(t(5)=5.66, p<.01$, Cohen's $d=3.77$ ) as is typical for final syllables in English. The average length for the trochaic tokens was $870 \mathrm{msec}(S D=54 \mathrm{msec})$ with an average second syllable onset of $339 \mathrm{msec}(S D=79 \mathrm{msec})$. For the iambic tokens, stressed syllables were significantly higher in pitch $(t(5)=17.55, p<.01$, Cohen's $d=6.14)$, higher in intensity $(t$ $(5)=5.65, p<.01$, Cohen's $d=3.81)$, and longer in duration, $(t(5)=6.43, p<.01$, Cohen's $d=4.66$ ) than unstressed syllables. The average length for the iambic tokens 
was $860 \mathrm{msec}(S D=91 \mathrm{msec})$ with an average second syllable onset of $250 \mathrm{msec}$ $(S D=91 \mathrm{msec})$.

The speaker recorded the following carrier $791 \mathrm{msec}$ phrase, for presentation at the beginning of each trial, "Where's the [target]?" Each target word was recorded in isolation and then presented embedded in the carrier phrase. The initial sentence with the target or distractor word, was followed by $750 \mathrm{msec}$ of silence and then one of three prompting sentences. The second prompt was an average of $1.39 \mathrm{sec}$ long and was either; "Can you find it?" for apple, baby, banana, and bunny, "Do you like it?" for chicken, dinosaur, kitten, and puppy, or "Do you see it?" for bottle, butterfly, flower, and teddy. The second prompts were varied to enhance infant engagement throughout the task. The trials were on average 7 sec long.

\section{Visual stimuli}

We used a split screen design, wherein infants saw the target object on one side and the distractor on the other side. The visual pairings of target and distractor objects were semi-random whereby the objects were divided into three groups and randomly paired within each group as shown in Table 2. There were six target objects: apple, baby, flower, kitten, puppy, and teddy. Each of these objects was shown twice, once while hearing the object's correct stressed label and once while hearing the object's mis-stressed label, making for a total of 12 stress test trials. The two presentations of each target object were always presented with different distractor objects, such that no stress test trials had the same object pairings. The following familiar distractors objects were shown with the target objects; banana, bottle, bunny, dinosaur, chicken, and butterfly.

In addition to the 12 stress test trials, there were six distractor trials that functioned as a control measure to ensure infants could successfully follow the procedure (i.e., locate the referent of a familiar word). The distractor trials were also used to assess whether infants were familiar with the labels of the chosen distractors. These trials displayed the following pairs of objects; banana with bottle, bunny with dinosaur, and chicken with butterfly. Each pairing was shown twice so that infants would be tested on their understanding of both object labels.

Trials were presented in a semi-randomized fashion. We randomly interspersed stress and distractor trials, such that trial type was not repeated more than three times in a row. Additionally, we ensured that the same distractors and targets were not

TABLE 1

Average Acoustic Measurements for Trochaic and lambic Stimuli (Standard Deviation in parentheses)

\begin{tabular}{lccc}
\hline & Pitch $(\mathrm{Hz})$ & Intensity $(\mathrm{dB})$ & Duration (msec) \\
\hline Trochaic & & & $205(103)$ \\
Stressed & $396(52)$ & $75(3.8)$ & $512(51)$ \\
Unstressed & $253(34)$ & $<.01$ & $<.01$ \\
$p$ Value & $<.001$ & $78(1.7)$ & $158(80)$ \\
Iambic & $224(6)$ & $84(1.2)$ & $584(102)$ \\
$\quad$ Unstressed & $293(15)$ & $<.01$ & $<.01$ \\
Stressed & $<.01$ & & \\
$p$ Value & & & \\
\hline
\end{tabular}


shown on consecutive trials. Presentation side of the target or labeled distractor was counterbalanced across participants.

\section{Apparatus}

The study was carried out in a small, dimly lit, sound-attenuated testing room. Each child was seated in a high-chair approximately two feet in front of a Tobii 1750 eye tracker. The stimuli were played on computer speakers at a volume of $65 \pm 5 \mathrm{~dB}$. Caregivers were seated next to their child and were given music played over Bose headphones during the procedure to ensure masking of the stimuli. The experimenter sat at a computer hidden from the infants' line of sight; however, the experimenter could view the infants through a camera. Calibration for eye-tracking was done through the program Clearview, while the study was conducted through E-prime software and Tobii eye-tracking hardware. Participants' eye movements were recorded in relation to specific areas of interest (AOIs), which were defined prior to data collection to correspond to the location of display objects. The areas of interest encompassed the objects themselves and did not include other areas of the screen (i.e., the background). Gaze data were logged every $20 \mathrm{msec}$, and fixations were defined as looks to the same image that lasted longer than $100 \mathrm{msec}$.

\section{Procedure}

Prior to testing, parents were asked to fill out the MacArthur Communicative Development Inventory short form (Fenson et al., 2000) to assess their infant's vocabulary development. Testing began with a calibration procedure during which infants were required to fixate on a duck as it moved to five different areas of the screen while a bell noise was played. Calibration was considered successful if infants calibrated to three of the five fixation points.

Following calibration, the test trials began. Each trial was proceeded by an attention-getter involving a silent, dynamic circle changing in color and shape. Once an infant was fixated on the screen, the experimenter pressed a designated button to terminate the attention-getter and begin the trial. Each trial began with the visual stimuli appearing, followed by the auditory prompts $3 \mathrm{sec}$ later. Stress test trials had a target object and distractor displayed while the target word was played. Distractor trials had two distractors displayed while one distractor was labeled. The entire procedure lasted approximately $5 \mathrm{~min}$.

TABLE 2

Visual Stimuli Pairing Groups for Test Trials

\begin{tabular}{lll}
\hline Group 1 & Group 2 & Group 3 \\
\hline Targets & & \\
Flower & Baby & Teddy \\
Kitten & Puppy & Apple \\
Distractors & & \\
Banana & Chicken & Butterfly \\
Bunny & Dinosaur & Bottle \\
\hline
\end{tabular}




\section{Coding and data preparation}

Analyses were calculated on eye gaze fixations starting at $200 \mathrm{msec}$ after syllable onset over a $2 \mathrm{sec}$ window. Beginning the analysis after syllable onset rather than word onset is more appropriate for the stimuli used here, since the mispronunciation of stress occurs over the course of a word and therefore might not be detected until after the beginning of the second syllable. Although $200 \mathrm{msec}$ is toward the lower minimum latency to initiate eye movements, prior studies have shown there to be no difference in analyses between $200 \mathrm{msec}$ up to $400 \mathrm{msec}$ after word onset (for review see Swingley \& Aslin, 2000).

Prior to exploring the effects of mis-stressing labels on infants' pattern of the fixation, we performed a preliminary examination of the data to ensure that infants had measurable fixations on at least three of the twelve stress test trials and at least a third of the total number of trials in the experiment. Three participants (noted above in "Participants") did not meet this criterion and were excluded from analysis. Since all infants successfully calibrated at the beginning of the study, the lack of fixation data throughout the study after calibration could be from loss of tracking validity or low attention. These three infants successfully sat through the experiment and were not fussy. All three infants fixated on the screen at the beginning of each trial (a requirement to begin a trial). For these reasons, we believe that the lack of fixation data for these infants arose from a loss of tracking validity; however, we cannot rule out that inattention may have inflated this loss. For this reason, the attrition of these infants was classified separately as "insufficient fixation data." We also examined how children performed on the distractor trials. There were three infants who only looked preferentially to the labeled object in one or two of the distractor trials, suggesting that these infants did not understand the procedure or did not know the labels for at least half of the distractor objects. These three infants were removed from the analysis, which left a final sample of 25 infants who were included in the analysis.

\section{RESULTS}

Recall that if infants have specified representations of lexical stress in their known words, then we would predict a greater proportion of fixations to the target referent when hearing correctly stressed labels versus hearing mis-stressed labels. To assess this prediction, we first examined the proportion of total fixations to target for the correctly stressed trials and the mis-stressed trials using a one-way analysis of variance. To calculate proportion of total fixations for each trial, we divided the total looking time to the target by the total looking to the target and distractor objects combined (i.e., $\mathrm{T} / \mathrm{T}+\mathrm{D})$. Infants' proportion of fixations to the target object was significantly above chance (.50) during the properly stressed trials $(M=.63, S D=.21), t$ $(24)=3.312, p=.004,95 \%$ CI $[.55, .71]$. However, infants' proportion of fixations did not differ from chance levels during the mis-stress trials $(M=.56, S D=.24), t$ $(24)=1.39, p=.177,95 \%$ CI $[.47, .66]$. These results suggest that infants successfully mapped properly stressed labels to the target objects but did not map the mis-stressed labels to either object.

To further examine how infants were processing the test labels, we conducted a growth curve analysis on the fixation data (Barr, 2008; Mirman, 2014; Mirman, Dixon, 
\& Magnuson, 2008; Mirman \& Magnuson, 2008). Growth curve models allow us to examine the dynamic nature of infants' looking behavior and provide more power than examining separate time bins. Our analysis used the lme4 package (Bates, Maechler, Bolker, \& Walker, 2015) and lmerTest package (Kuznetsova, Brockhoff, \& Christensen, 2014) in R Studio Version 0.99.491. Time was modeled using orthogonal polynomials (linear, quadratic, and cubic). Trial effects and interactions were modeled as fixed effects, while subject factors were modeled as random effects with random slopes, intercepts, and interactions between subjects and time for each of the polynomial orders. In this analysis, we measured the proportional looking to each object for each time sample (i.e., 20-msec bin) using the time sample bin length as the denominator for each time point score (i.e., looking to an object/interval time length). As a result, a value of 1 for looking to the target object indicated that an infant fixated only on the target during that interval, and a value of 0 indicated that an infant did not fixate on the target during that interval (i.e., fixated on the distractor, fixated on the screen's background or looking off-screen). Average proportional looking times were calculated for each time sample bin for the target object and for the distractor object. We then calculated a difference score between the fixations to the target and distractor to create a single score (i.e., target-distractor). We used a difference score for the following reasons: Proportional measures that use the bin length as a denominator are more sensitive to lack of attention and can present a more accurate measure of infants' ability to fixate on the correct object. The previous analysis which used proportion of total looks can produce extreme proportional values even if looking time was relatively low. This can, in turn, increase the overall variance and make it more difficult to detect true differences in fixations among trial types. For our growth curve model, we sought to use a measure that was more sensitive to low looking times but was less susceptible to possibly inaccurate extreme scores. Unlike the previous analysis which examined looking over the entire trial, in our growth curve model we were interested in the speed and degree of fixation changes across the experiment and so wanted a more stable measure that would accurately account for low looking.

Figure 1 displays the difference scores between the proportional fixations to the target and distractor. When presented with mis-stressed labels, infants had an average difference score of .06 over the entire analysis window (Estimate $=0.05 ; S E=0.02$; $t=2.93 ; p<.05)$. When presented with properly stressed labels, this difference score rose to .12 (Estimate $=0.06 ; S E=0.03 ; t=2.45 ; p<.05)$. This suggests that in both trial types infants fixated to the target significantly more than the distractor object. Additionally, there was a significant interaction between word stress and linear time (Estimate $=0.18 ; S E=0.05 ; t=3.75 ; p<.001)$ and between word stress and quadratic time (Estimate $=-0.12 ; S E=0.05 ; t=-2.53 ; p<.05$ ). The significant positive linear interaction suggests that the overall slope of infants' looking behavior in the properly stressed word trials was steeper than in the mis-stressed trials, whereas the significant negative quadratic effect suggests that with properly stressed words there was less of a bend in the fixation curve compared to the trials which had mis-stressed words. The cubic term did not significantly interact with word stress (Estimate $=0.05 ; S E=0.05$; $t=1.00 ; p>.05)$. These results indicate infants looked to the target object faster and increased their looking to the target throughout the trial upon hearing properly stressed labels, whereas infants were slower to fixate on the target and their looking behavior toward the target plateaued upon hearing the mis-stressed labels. 
Finally, we examined whether infants' sensitivity to the stress patterns of familiar words was related to overall vocabulary size by comparing nested models. Infants' mean comprehension score was $56.58(S D=16.79)$, and mean production score was $15.64(S D=14.72)$. These scores for comprehension and production correspond to the 60th and 50th percentiles, respectively (Fenson et al., 2000). See Figure 2 for complete CDI scores. In no case did comprehension vocabulary effect the intercept $\left(\chi^{2}(1)=.18\right.$, $p=.67)$ or interact with word stress $\left(\chi^{2}(2)=.67, p=.71\right)$. Similarly, infants' productive vocabulary did not affect the intercept $\left(\chi^{2}(1)=3.35, p=.07\right)$ or interact with word $\operatorname{stress}\left(\chi^{2}(2)=3.42, p=.18\right)$.

\section{DISCUSSION}

The results of Experiment 1 indicate that infants are sensitive to stress patterns of familiar words. The fixation patterns suggest that infants are less reliable and slower in their mappings of familiar target labels when they are mis-stressed. Infants' proportion of total looking indicated that they did not map the mis-stressed label to the target; however, infants' proportional of looking over trial length in our growth curve analysis suggested that infants considered the mis-stressed form as a possible label for the target object. The time-course analysis revealed further differences in how infants were processing mis-stressed labels and properly stressed labels. Compared with the properly stressed labels, infants were slower to fixate on the labeled object and less reliable in fixating on the labeled object when labels were mis-stressed. Based on these analyses, we cannot conclusively state whether infants interpreted the mis-stressed words as possible labels for the target object.

These findings support the claim that 17-month-old infants are encoding lexical stress in their familiar word representations. Mis-stressed words are not as easily linked with target objects when infants view a target with a familiar distractor, but they do not clearly treat a mis-stressed label as a label for the familiar distractor. This result

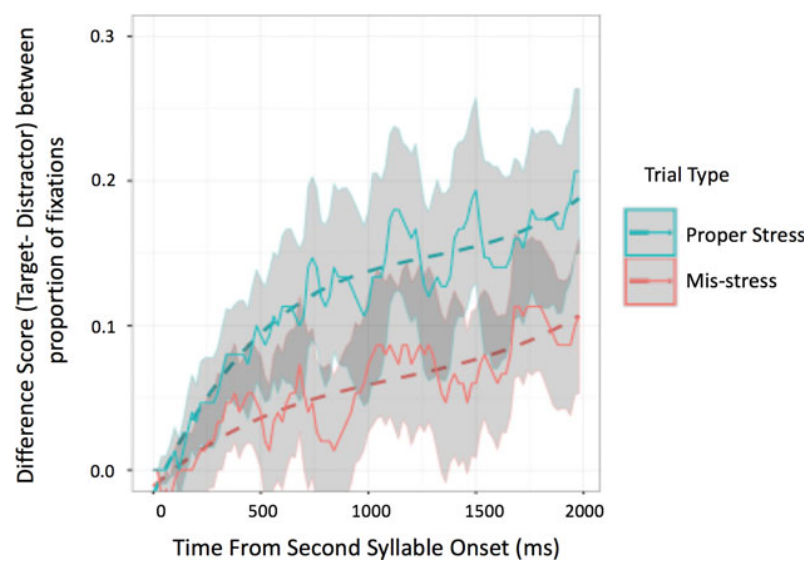

Figure 1 Difference scores between the proportion fixations at target minus distractor relative to the total trial length when infants were presented with two familiar objects. Ribbons display the standard error of the mean and dotted lines showing the model fits. 


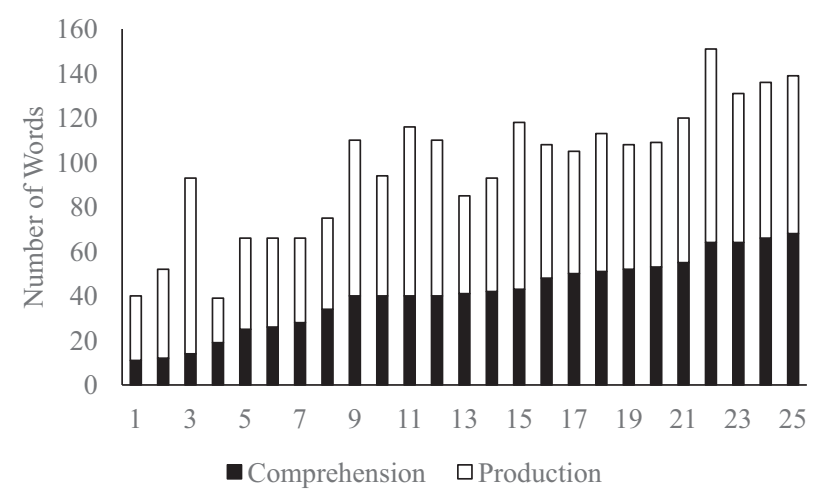

Figure 2 CDI scores for participants in Experiment 1.

leaves open the possibility that infants interpreted the mis-stressed word as a novel word but that when given two familiar objects, they are uncertain as to the interpretation. If presented with a novel distractor object, infants may have mapped the misstressed label to the novel object. Our second experiment further explores this possibility and probes the nature and extent to which infants encode lexical stress in their familiar words and appreciate the nonproductive nature of lexical stress.

\section{EXPERIMENT 2}

In Experiment 2, we asked whether infants treat a mis-stressed familiar label as a novel word and thus map it to a unique referent. Previous studies have shown that younger infants will map two novel words differing in only their stress patterns to separate novel objects (Curtin, 2010). This work supports the claim that infants might encode a familiar word presented with a different stress pattern as a novel word. To explore this possibility, 17-month-olds heard the mis-stressed word while viewing a familiar object paired with a novel object. The task here was similar in design to disambiguation studies, wherein children are presented with a novel word while viewing a pair of objects, one of which is familiar and one of which is novel. Research on disambiguation tasks has found that children as young as $16-18$ months who view a familiar object paired with a novel objet will choose the novel object as the referent for a novel word (Byers-Heinlein \& Werker, 2009; Graham, Poulin-Dubois, \& Baker, 1998; Halberda, 2003; Markman, Wasow, \& Hansen, 2003). If word stress is strongly encoded in familiar word representations, a different stress pattern could signal to infants a new potential word to attach to the novel object. This would suggest that infants treat lexical stress in familiar words as a key component of their representation that distinguishes that word from others. If, however, infants map a mis-stressed label to the target object when provided a possible novel referent, this would suggest that infants do not treat mis-stressed familiar words as new words and appreciate the nonproductive nature of lexical stress.

\section{Participants}

Twenty-five 17-month-old infants $(M=17$ months 16 days, $S D=8$ days, range: 17 months 5 days to 17 months 28 days) were included in the final sample. Infants 
came from homes where English was the primary spoken language. An additional 13 infants were tested; however, they were removed due to fussiness $(N=7)$, parental inference $(N=3)$, experimenter error $(N=3)$. As in Experiment 1 , infants were also excluded post hoc on the basis of insufficient fixation data $(N=6)$.

\section{Auditory stimuli}

The auditory labels provided to the infants were identical to those used in Experiment 1.

\section{Visual stimuli}

The objects were presented in an identical manner as in Experiment 1 with one exception: On the stress trials, infants were presented with a novel object and a familiar object (see Figure 3 for example novel objects). On six trials, infants viewed two familiar objects. These trials were identical to the distractor trials presented in Experiment 1 and were used to establish whether infants understood the procedures of the study. As in the first experiment, trials were presented in a semi-randomized fashion and infants were randomly assigned to each presentation order.

\section{Apparatus}

Same as in Experiment 1.

\section{Procedure}

Same procedure as used in the first experiment.

\section{Coding}

Coding was conducted in the same manner as Experiment 1. There were six infants (noted above in "Participants") in this experiment who had insufficient fixation data. We used the same criteria for exclusion as in Experiment 1. All infants preferentially looked at the target on at least three of the six of the familiar object pairing trials and appeared to understand the task. Therefore, no infants were excluded on the basis of not knowing the familiar objects. The final sample included 25 participants.
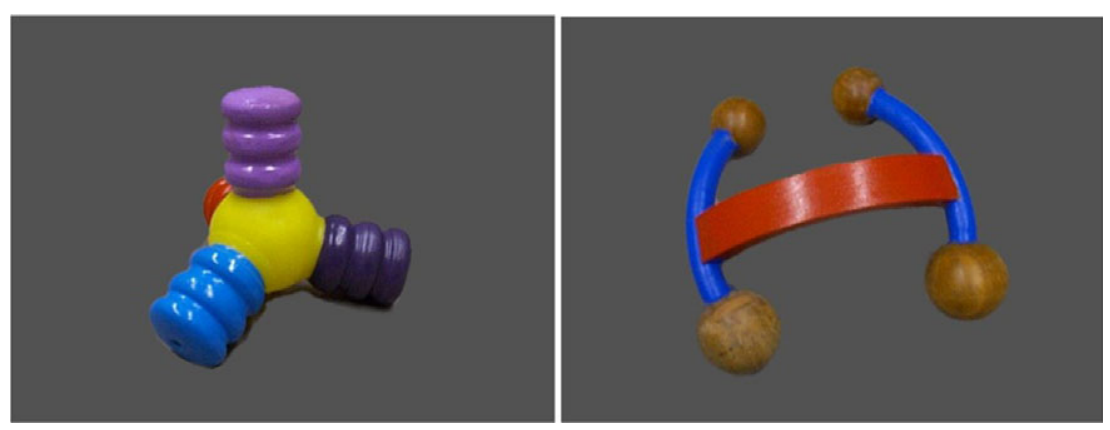

Figure 3 Example of novel toy stimuli used in the Novel Distractor Condition. 


\section{RESULTS}

During the properly stressed trials, infants fixated on the target significantly more than chance $(.50 ; M=.73, S D=.22), t(24)=5.05, p<.01,95 \%$ CI $[.64, .81]$. However, in the mis-stressed trials, infants' fixations did not differ from chance $(M=.52$, $S D=.06), t(24)=0.27, p=.79,95 \%$ CI $[.41, .62]$. These results suggest that while infants successfully mapped the properly stressed labels to the target objects, they did not map these mis-stressed labels to either object on screen.

We then ran a growth curve analysis with the same parameters as in Experiment 1 and again using a difference score between the target and distractor proportional looking times (of entire trial or bin length). Figure 4 shows the difference scores between target and distractor with our model superimposed. There was a significant increase in looking at the target in the correct stressed word trials $(M=.13$, Estimate $=0.13$; $S E=0.004 ; t=3.58 ; p<.05)$. The model intercept indicated the overall difference score for the mis-stressed trials was -.006 and was not different from zero (Estimate $=-.006 ; S E=.03 ; t=-.24 ; p>.05$ ). Over the entire analysis window, infants fixated toward the target when hearing properly stressed words but did not fixate toward the target when hearing the mis-stressed words. Additionally, there were significant interactions between the linear term and word stress (Estimate $=0.48 ; S E=0.05$; $t=10.57 ; p<.001$ ), between the quadratic term and word stress (Estimate $=-.06$; $S E=0.05 ; t=-12.88 ; p<.001)$, and between the cubic term and word stress (Estimate $=-.38 ; S E=0.05 ; t=-8.41 ; p>.001)$. This suggests that when hearing properly stressed labels, infants' looking toward the target had a more positive slope and there was less of a change in fixations from the target toward the distractor. In contrast, when hearing mis-stressed labels, infants' looking to the target had a slightly negative slope and the looking toward the distractor increased at the beginning of the analysis window. Additionally, with mis-stressed labels infants' fixations fluctuated between the target and distractor more so than in the correct stressed trials, suggesting an overall less stable mapping of the mis-stressed labels.

We further examined whether infants' mappings of the familiar words were related to overall vocabulary development by comparing nested models. Our sample's mean comprehension score was $45.16(S D=12.50)$, and mean production score was 14.72 $(S D=10.76)$. These scores for comprehension and production correspond to the 55th and 48th percentiles, respectively (Fenson et al., 2000). See Figure 5 for complete CDI scores. The difference score between infants' fixations to the target and distractor proportioned by the total trial length, infant comprehension vocabulary did not affect the intercept $\left(\chi^{2}(1)=.11, p=.74\right)$ or interact with word stress $\left(\chi^{2}(2)=.21, p=.90\right)$. Additionally, infants' productive vocabulary did not affect the intercept $\left(\chi^{2}(1)=3.20\right.$, $p=.07)$ or interact with word stress $\left(\chi^{2}(2)=5.24, p=.07\right)$.

\section{DISCUSSION}

These results support the findings of the first experiment. Infants' fixation patterns when hearing the correctly stressed labels were different than when hearing mis-stressed labels. When infants were shown target images paired with novel images, their proportion of fixations to target were reliably above chance when hearing the properly stressed labels but not above chance when hearing the mis-stressed labels. This was 


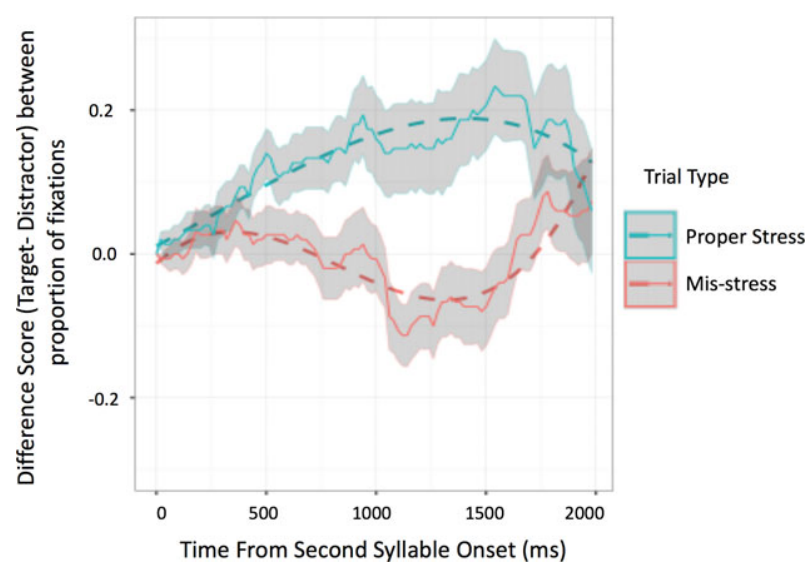

Figure 4 Difference scores between the proportion fixations at target and distractor relative to the total trial length when infants were presented with one familiar object and one novel object. Ribbons display the standard error of the mean and dotted lines showing the model fits.

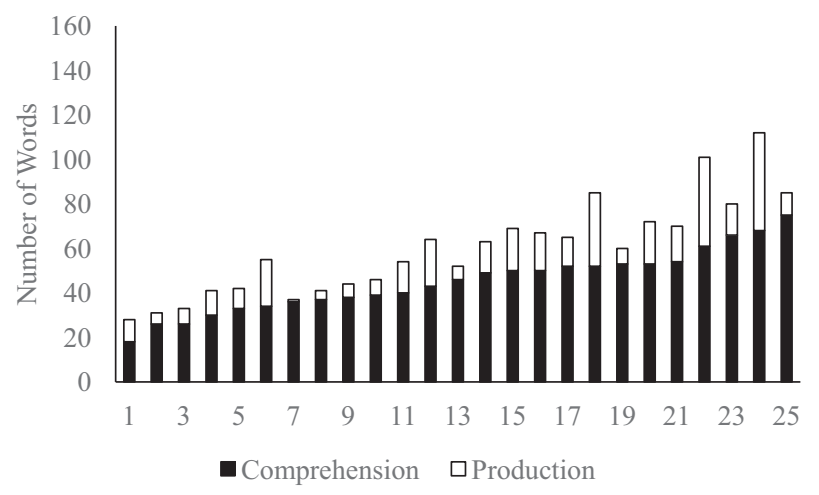

Figure 5 CDI scores for participants in Experiment 2.

consistent with the proportional looking times of total bin length used in the timecourse analysis, which revealed that infants only reliably mapped the target word to the correct object when given the label that was properly stressed. When infants were given a mis-stressed label, they were slower to fixate on the target and fluctuated between fixations to the target and distractor. Together, these results suggest that infants are sensitive to the lexical stress of their familiar word representations. Infants have finely, detailed familiar word representations that include information about the stress of the word. Lexical stress of a familiar word may be an important feature for infants but infants do not treat a mis-stressed word as a novel object label.

\section{GENERAL DISCUSSION}

We explored the extent to which lexical stress impacts 17-month-olds' recognition of familiar words. When infants were presented with mis-stressed familiar words, their 
proportion of fixations to the target object were lower and in one experiment did not reliably differ from chance-level responding, compared to when they were given properly stressed labels. These results suggest that infants are less reliable in their mapping of mis-stressed familiar words regardless of whether the distractors are familiar or novel objects. When viewing the target paired with a familiar distractor, time-course analyses revealed that infants were slower to fixate on the target and showed a lower fixation rate to target with mis-stressed labels than with properly stressed labels. When infants viewed the target object paired with a novel distractor, infants mapped the properly stressed label to the target but did not appear to reliably map the mis-stressed label to either the target object or distractor object. Together these results suggest that infants have highly detailed representations of their familiar words and that they may use word stress in their recognition of familiar words.

Our results are consistent with previous literature demonstrating that young infants have finely detailed representations of novel and familiar words (for review see Bailey \& Plunkett, 2002). By 14-18 months, infants map novel segmental-minimal pairs and detect consonant and vowel mispronunciations of familiar words (Bailey \& Plunkett, 2002; Fennell \& Werker, 2003; Swingley \& Aslin, 2000, 2002). Twelve-month-olds encode stress in their novel word representations (Curtin, 2009, 2010, 2011), and word stress is stored in familiar word representations by Dutch toddlers (Van Alphen et al., 2007). Our work here further expands on this previous research and provides further support that infants encode stress in their familiar word representations. Not only are infants storing lexical stress in their novel word representations but they continue to store this information in known words even though this information beyond what is needed to distinguish the majority of words from one another.

These findings from Experiment 1 challenge the developmental hypothesis that a word learner only needs to have enough representational information to contrast the words within their known lexicon (for review see Bailey \& Plunkett, 2002). This hypothesis predicts that older children and children with higher vocabularies would have richer representations of their words. However, the current study did not find any effects of vocabulary, receptive, or expressive, on the ability to detect the mispronunciations of stress. Our findings are consistent with previous work in familiar word segmental studies, which have also found there to be no effect of vocabulary or of age on infants' ability to detect mispronunciations (Bailey \& Plunkett, 2002; Swingley \& Aslin, 2000). Although we cannot strongly argue against the developmental hypothesis, our study does suggest that it is not necessary for infants to have a high vocabulary or to have high density lexical neighborhoods to detect mis-stressing of familiar words.

Consistent with previous findings, our results from Experiment 1 further demonstrated that infants' identification of the correct referent occurred across a continuum (Swingley \& Aslin, 2000, 2002). Infants' word representations appeared to be flexible when in the presence of two familiar objects. The time-course analysis which used proportional looking of bin length found that when infants were presented with the incorrect stress pattern, they still looked to the target more than the distractor and with this measure infants did eventually map the label to the correct object when given familiar distractors. However, we did find that with the mis-stressed words infants were fixating later and less reliably to the target in interval analyses, indicating that there was lower activation of the target. This finding of a gradient of activation has been found in other mispronunciation studies using familiar words (Swingley \& Aslin, 2000, 2002) as well as in disambiguation studies (Bion, Borovsky, \& Fernald, 2013). 
In Experiment 2, when infants heard a mis-stressed label in the presence of a novel object, they increased their looking toward the novel object during part of the test trial but overall looked equally at the novel and target. Infants did not show evidence of a disambiguation bias or mutual exclusivity by mapping the mis-stressed word to the novel distractor reliably. These results are consistent with other work showing that 18month-olds are still developing disambiguation skills and can be unreliable in their mappings (Bion et al., 2013). Although there are studies which have found disambiguation effects in 17- and 18-month-olds (e.g., Byers-Heinlein \& Werker, 2009; Halberda, 2003), many of these studies have used blocked designs that first presented infants with pairs of known objects before testing for any disambiguation effects. In procedures more similar to ours (which did not use a blocked presentation), researchers have found disambiguation results similar to ours (Mather \& Plunkett, 2009, 2010, 2011). In these studies by Mather and Plunkett, infants were presented with two objects, one familiar and one novel, while hearing a novel label embedded in a carrier phrase (e.g., "Look! Look at the meb! Look! Meb!"). Although each of these experiments had at least one novel label trial and control trial, they were not presented in a block-like manner with control trials always appearing at the beginning of the experiment.

Similar to our results of Experiment 2, Mather and Plunkett (2009, 2010, 2011) found disambiguation effects only during some segments of trials. Infants' fixations upon hearing a novel label fluctuated between the familiar and novel object with only parts of trials indicating that infants were mapping to the novel object. For instance, in Mather and Plunkett's (2010) study, 10-month-olds showed disambiguation (i.e., looking more to the novel object) between $7.5 \mathrm{sec}$ to $10 \mathrm{sec}$ into the trial. Similarly, in Mather and Plunkett's (2010) study, 22.5-month-olds showed a preference for the novel object from $1.5 \mathrm{~s}$ to $4.5 \mathrm{~s}$ into the trial but otherwise appear to have no preference. Our results appear to replicate these effects. Similar to some studies, our infants did not interpret the mispronounced labels as labels for the target objects (e.g., ByersHeinlein \& Werker, 2009; Halberda, 2003); however, our time-course analysis reveals that while infants in our study appeared to entertain the novel object as the possible referent, they did not settle on that form.

The use of preceding familiar object trials, however, is not required for infants to show disambiguation. In one study, when 16- to 22 -month-olds were presented with two familiar objects and a third novel object, they interpreted a novel label as referring to the novel object (Graham et al., 1998). Age did not appear to affect performance; however, those infants with higher productive vocabularies were more likely to interpret the novel word as a label for the novel object. Thus infants as young as 16 months of age appear to be able to disambiguate the referent of a novel word. Although our studies presented here did not find vocabulary effects, this work raises the possibility that with a sample that had a larger range of vocabularies and with a more sensitive task, our infants would have demonstrated disambiguation effects in Experiment 2. However, it also remains possible that infants treat familiar words with consonant mispronunciations as novel words, but will not fully commit to treating familiar words with lexical stress mispronunciations as novel words.

Infants reluctance to map a mis-stressed familiar word may have been as a result of our focus on object labels. As mentioned, the majority of disyllabic English nouns are trochaic, while most bisyllabic verbs are iambic (Kelly \& Bock, 1988). This could potentially explain why there was reluctance to map the iambic (mis-stressed) form 
onto a novel object. When a child encounters a novel trochaic word, they can infer that the word is a probable label for a noun rather than a verb and can narrow their focus of potential referents to help with faster word mapping. Conversely, when encountering a novel iambic word, an infant could narrow their focus to actions. Indeed, infants as young as 16 months are biased to accept only iambic labels for actions and are unable to map the same labels presented with trochaic stress (Campbell et al., 2018; Curtin et al., 2012). By linking a novel word into its grammatical category, word learners can more easily produce the multiple morphological forms by generalizing what is typical for that category (Gervain \& Werker, 2008). It is possible that infants' failure to map the mis-stressed (iambic) familiar word may have been influenced by an implicit preference to map iambic labels as action labels or to use word stress to distinguish grammatical category membership but this experiment limited infants to only novel object-label referents and did not support alternative interpretations. Specifically, the objects presented to infants in our study were stationary and thus an interpretation of the novel labels as naming actions was not supported by the experiment. Faced with only novel objects, infants did not map the mis-stressed (iambic) familiar word to a novel object.

Although our experiments support the claim that infants store lexical stress in their familiar word representations and will not interpret a mis-stressed word as a novel label, these results may only be true for familiar trochaic words. Research from adult studies has shown that for iambic labels adults often need an additional cue of vowel reduction to detect mis-stressed items (Cutler \& Clifton, 1984). Moreover, adults often can only detect segmental mispronunciations that occur in stressed syllables (Mattys \& Samuel, 1997, 2000). Infant research has also shown that it is more difficult to detect segmental mispronunciations in unstressed syllables suggesting that infants are potentially encoding stressed and unstressed syllables differently (Floccia, Nazzi, Austin, Arreckx, and Goslin (2011); Halle \& de Boysson-Bardies, 1996; Vihman et al., 2004). Given this previous research, our findings may not generalize to mis-stressing of iambic labels, and it would be of interest to further explore this possibility.

Research with adults raises the possibility that infants may not only be attending to lexical stress to detect mispronunciations. As discussed earlier, adults are more sensitive to mis-stressed words when the change in stress is accompanied by a vowel reduction (Cutler \& Clifton, 1984), and vowel reduction is one of the strongest cues to lexical stress for adult English speakers (Zhang \& Francis, 2010). While our stimuli maintained the vowel quality as much as possible in a natural production, it is possible that infants in our sample were attending to subtle changes in the vowel quality of the familiar words (See Table 3 for vowel measurements for each test word). Although infant studies have not directly examined this question, studies have demonstrated that toddlers are sensitive to vowel mispronunciations of familiar words (Swingley, 2016). Research on infants' representations of newly learned words has further suggested that 15-month-olds are sensitive vowels such that they can learn two words that differ only in a single vowel feature, such as height (Curtin, Fennell, \& Escudero, 2009). Moreover, work using novel words has shown that when 18-month-olds can detect changes in vowel quality in a habituation task (Dietrich, Swingley, \& Werker, 2007). Although it has not been shown that infants can attend to vowel quality changes in familiar words, past research has demonstrated that infants attend closely to changes in vowels and specifically in vowel quality in newly learned words. Infants in our study may have 
TABLE 3

Average F1 and F2 Measurements for Trochaic and lambic Stimuli

\begin{tabular}{|c|c|c|c|c|}
\hline & \multicolumn{2}{|c|}{ Syllable 1} & \multicolumn{2}{|c|}{ Syllable 2} \\
\hline & $F 1$ & $F 2$ & $F 1$ & $F 2$ \\
\hline /'æp $1 / /$ & 876 & 1,613 & 506 & 1,147 \\
\hline /æ’p $\varepsilon 1 /$ & 989 & 1,688 & 598 & 1,144 \\
\hline /'beybi// & 508 & 2,517 & 354 & 2,824 \\
\hline /bey'bi/ & 470 & 2,743 & 527 & 3,073 \\
\hline /'flowa व / & 479 & 832 & 556 & 1,169 \\
\hline /flow’o व / & 385 & 875 & 563 & 1,142 \\
\hline /'kitən/*' & 489 & 2,521 & 423 & 1,911 \\
\hline /kı'tən/ & 420 & 2,261 & 433 & 2,117 \\
\hline /'p $\wedge \mathrm{pi} /$ & 520 & 1,416 & 239 & 2,976 \\
\hline /p^’pi/ & 317 & 1,487 & 391 & 3,366 \\
\hline /'tedi/ & 524 & 2,451 & 270 & 2,863 \\
\hline$/ \mathrm{t} \varepsilon^{\prime} \mathrm{di} /$ & 402 & 2,315 & 284 & 2,964 \\
\hline
\end{tabular}

*Kitten was produced without a flap in order to preserve segmental quality across stress types. All tokens were produced in infant-directed speech.

been attending to subtle vowel quality changes to detect the mispronunciations of familiar words. Attending to lexical stress and vowel quality would help infants to further distinguish a correctly pronounced word from an incorrectly stressed word. Further research is needed to tease apart how infants may use these two cues when processing familiar words.

In summary, our research suggests that 17-month-old infants have difficulty in recognizing familiar trochaic words when they are presented with the incorrect stress pattern. These results suggest that familiar representations include information of word stress. A correctly stressed word best matches the representation and facilitates faster and more reliable identification of the target whereas mis-stressed words appear to hinder identification of the target. Further, infants do not map a mis-stressed form in the same manner as a properly stressed form. Whether infants map a mis-stressed iambic form onto a novel object is unclear and is a question in need of further research. Our research in conjunction with previous literature provides support for rich representations that include stress information and sophisticated word recognition abilities.

\section{ACKNOWLEDGMENTS}

This research was supported by a Natural Sciences and Engineering Research Council of Canada (NSERC: \#327319-2012) grant awarded to Suzanne Curtin and a Natural Sciences and Engineering Research Council of Canada (NSERC: \#194530-2011) grant awarded to Susan Graham. There are no conflicts of interest, real or perceived, in the design, collection, analysis, and interpretation of data. We would like to thank Jared Berman and Patrick Mihalicz and the members of the Speech Development Lab for their support with this research. This research would not be possible without the generous time and support of the families and their infants who participated. 


\section{REFERENCES}

Bailey, T.M., \& Plunkett, K. (2002). Phonological specificity in early words. Cognitive Development, 17, 1265-1282.

Barr, D.J. (2008). Analyzing 'visual word' eyetracking data using multilevel logistic regression. Journal of Memory and Language, 59, 457-474.

Bates, D., Maechler, M., Bolker, B., \& Walker, S. (2015). Fitting Linear Mixed-Effects Models Using lme4. Journal of Statistical Software, 67, 1-48.

Bion, R.A.H., Borovsky, A., \& Fernald, A. (2013). Fast mapping, slow learning: Disambiguation of novel word-object mappings in relation to vocabulary learning at 18, 24, and 30 months. Cognition, 126, 39-53.

Byers-Heinlein, K., \& Werker, J. (2009). Monolingual, bilingual, trilingual: Infants' language experience influences the development of a word- learning heuristic. Developmental Science, 12, 815-823.

Campbell, J., Mihalicz, P., Theissen, E., \& Curtin, S. (2018). Does Lexical Stress Influence 17-month-olds' Mapping of Verbs and Nouns? Developmental Psychology, 54, 621-630.

Chomsky, N., \& Halle, M. (1968). The sound pattern of English. New York: Harper and Row. xiv, p. 470 Reprinted 1991, Boston: MIT Press.

Curtin, S. (2009). Twelve-month-olds learn novel word-object pairs differing only in stress pattern. Journal of Child Language, 36, 1157-1165.

Curtin, S. (2010). Young infants encode lexical stress in newly encountered words. Journal of Experimental Child Psychology, 105, 376-385.

Curtin, S. (2011). Do newly formed word representations encode non-criterial information? Journal of Child Language, 38, 904-917.

Curtin, S., Campbell, J., \& Hufnagle, D.H. (2012). Mapping Novel Labels to Actions: How the rhythm of words guides infants' learning. Journal of Experimental Child Psychology, 112, 127-140.

Curtin, S., Fennell, C., \& Escudero, P. (2009). Weighting of vowel cues explains patterns of word-object associative learning. Developmental Science, 12, 725-731.

Curtin, S., Mintz, T.H., \& Christiansen, M.H. (2005). Stress changes the representational landscape: Evidence from word segmentation. Cognition, 96, 233-262.

Curtin, S., \& Zamuner, T.S. (2014). Understanding the developing sound system: Interactions between sounds and words. Wiley Interdisciplinary Reviews: Cognitive Science, 5, 589-602.

Cutler, A. (2004). Lexical Stress. In D.B. Pisoni, \& R.E. Remez (Eds.), The handbook of speech perception (pp. 264-289). Oxford, UK: Blackwell Publishing.

Cutler, A., \& Clifton, C. (1984). The use of prosodic information in word recognition. In H. Bouma, \& D.G. Bouwhuis (Eds.), Attention and performance X: Control of language processes (pp. 183-196). Hillsdale, NJ: Erlbaum.

Dietrich, C., Swingley, D., \& Werker, J.F. (2007). Native language governs interpretation of salient speech sound differences at 18 months. Proceedings of the National Academy of Sciences of the United States of America, 104, 16027-16031.

Echols, C.H., Crowhurst, M.J., \& Childers, J. (1997). The perception of rhythmic units in speech by infants and adults. Journal of Memory and Language, 36, 202-225.

Fennell, C.T., \& Werker, J.F. (2003). Early word learners' ability to access phonetic detail in well-known words. Language and Speech, 46, 245-264.

Fenson, L., Pethick, S., Renda, C., Cox, J.L., Dale, P.S., \& Reznick, J.S. (2000). Short-form versions of the MacArthur communicative development inventories. Applied Psycholinguistics, 21(1), 95-116.

Floccia, C., Nazzi, T., Austin, K., Arreckx, F., \& Goslin, J. (2011). Lexical stress and phonetic processing in word learning in 20- to 24- month- old English-learning children. Developmental Science, 14, $602-613$.

Gervain, J., \& Werker, J.F. (2008). How infant speech perception contributes to language acquisition. Language and Linguistics Compass, 2, 1149-1170.

Graham, S.A., Poulin-Dubois, D., \& Baker, R.K. (1998). Infants' disambiguation of novel object words. First Language, 18, 149-164.

Halberda, J. (2003). The development of a word-learning strategy. Cognition, 87, 23-34.

Halle, M. (1998). The stress of English words: 1968-1998. Linguistic Inquiry, 29, 539-568.

Halle, P.A., \& de Boysson-Bardies, B. (1996). The format of representation of recognized words in infants' early receptive lexicon. Infant Behaviour and Development, 19, 463-481.

Hayes, B. (1995). Metrical stress theory: Principles and case studies. University of Chicago Press.

Johnson, E.K., \& Jusczyk, P.W. (2001). Word segmentation by 8-month-olds: When speech cues count more than statistics. Journal of Memory and Language, 44, 548-567. 
Jusczyk, P.W., Cutler, A., \& Redanz, N.J. (1993). Infants' preference for the predominant stress patterns of English words. Child Development, 64, 675-687.

Kelly, M.H., \& Bock, J.K. (1988). Stress in time. Journal of Experimental Psychology: Human Perception and Performance, 14, 389-403.

Kuznetsova, A., Brockhoff, P., \& Christensen, R. (2014). ImerTest: Tests in Linear Mixed Effects Models. R package version 2.0-20. Retrieved from http://CRAN.R-project.org/package $=$ lmerTest

Lahiri, A., \& Marslen-Wilson, W.D. (1991). The mental representation of lexical form: A phonological approach to the mental lexicon. Cognition, 38, 245-294.

MacKenzie, H., Curtin, S., \& Graham, S. A. (2012). 12-month-olds' phonotactic knowledge guides their word-object mappings. Child Development, 83, 1129-1136.

MacKenzie, H., Graham, S., \& Curtin, S. (2011). Twelve-month-olds privilege words over other linguistic sounds in an associative learning task. Developmental Science, 14, 399-410.

Markman, E.M., Wasow, J.L., \& Hansen, M.B. (2003). Use of the mutual exclusivity assumption by young word learners. Cognitive Psychology, 47, 241-275.

Mather, E., \& Plunkett, K. (2009). Learning words over time: The role of stimulus repetition in mutual exclusivity. Infancy, 14, 60-76.

Mather, E., \& Plunkett, K. (2010). Novel labels support 10-month-olds' attention to novel objects. Journal of Experimental Child Psychology, 105, 232-242.

Mather, E., \& Plunkett, K. (2011). Mutual exclusivity and phonological novel constrain word learning at 16 months. Journal of Child Language, 38, 933-950.

Mattys, S.L., \& Samuel, A.G. (1997). How lexical stress affects speech segmentation and interactivity evidence from the migration paradigm. Journal of Memory and Language, 36, 87-116.

Mattys, S.L., \& Samuel, A.G. (2000). Implications of stress-pattern differences in spoken-word recognition. Journal of Memory and Language, 42, 571-596.

Mirman, D. (2014). Growth curve analysis and visualization using R. Boca Raton, FL: Chapman \& Hall/CRC.

Mirman, D., Dixon, J.A., \& Magnuson, J.S. (2008). Statistical and computational models of the visual word paradigm: Growth curves and individual differences. Journal of Memory and Language, 59, 475-494.

Mirman, D., \& Magnuson, J.S. (2008). Attractor dynamics and semantic neighborhood density: Processing is slowed by near neighbors and speeded by distant neighbors. Journal of Experimental Psychology: Learning, Memory, and Cognition, 34, 65-79.

Polka, L., \& Sundara, M. (2003). Word segmentation in monolingual and bilingual infant learners of English and French. In M.J. Solé, D. Recasens \& J. Romero (Eds.), Proceedings of the 15th International Congress of Phonetic Sciences, Barcelona (pp. 1021-1024).

Polka, L., Sundara, M., \& Blue, S. (2002). The role of language experience in word segmentation: A comparison of English, French, and bilingual infants. Paper presented at the 143rd Meeting of the Acoustical Society of America: Special Session in Memory of Peter Jusczyk, Pittsburgh, Pennsylvania.

Studdert-Kennedy, M. (1976). Speech perception. In N.J. Lass (Ed.), Contemporary issues in experimental phonetics (pp. 243-293). New York: Academic Press.

Swingley, D. (2016). Two-year-olds interpret novel phonological neighbors as familiar words. Developmental Psychology, 52, 1011.

Swingley, D., \& Aslin, R.N. (2000). Spoken word recognition and lexical representation in very young children. Cognition, 76, 147-166.

Swingley, D., \& Aslin, R.N. (2002). Lexical neighborhoods and the word-form representations of 14-montholds. Psychological Science, 13, 480-484.

Thiessen, E.D., \& Saffran, J.R. (2003). When cues collide: Use of stress and statistical cues to word boundaries by 7- to 9-month-old infants. Developmental Psychology, 39, 706-716.

van Alphen, P.M., de Bree, E., Fikkert, P., \& Wijnen, F. (2007). The role of metrical stress in comprehension and production of Dutch children at risk of dyslexia. In Proceedings of Interspeech 2007 (pp. 2313-2316). Adelaide, SA, Australia: Causal Productions.

Vihman, M.M., Nakai, S., DePaolis, R., \& Halle, P. (2004). The role of accentual pattern in early lexical representation. Journal of Memory and Language, 50, 336-353.

Zhang, Y., \& Francis, A. (2010). The weighting of vowel quality in native and non-native listeners' perception of English lexical stress. Journal of Phonetics, 38, 260-271. 